35 Phone: $+1-574-631-3327$

36 Fax: +1-574-631-2144

37

38

Glen L. Niebur, Ph. D.

Notre Dame, IN 46556

Email: gniebur@nd.edu

\section{Comparison of Solid and Fluid Constitutive Models of Bone Marrow During Trabecular Bone Compression}

\author{
Thomas A. Metzger and Glen L. Niebur
}

Tissue Mechanics Laboratory, Bioengineering Graduate Program, University of Notre Dame

Technical Brief

June 15, 2016

Running title: Marrow Constitutive Models

Submitted to: J. Biomechanics

Word Count: 1,975 (Introduction to acknowledgements)

Address Correspondence to:

Department of Aerospace and Mechanical Engineering

Bioengineering Graduate Program

University of Notre Dame 


\section{Abstract}

40 The mechanical environment and mechanobiology of bone marrow may play essential roles in

41 bone adaptation, cancer metastasis, and immune cell regulation. However, the location of

42 marrow within the trabecular pore space complicates experimental measurement of marrow

43 mechanics. Computational models provide a means to assess the shear stress and pressure in the

44 marrow during physiological loading, but they rely on accurate inputs for the marrow and the

45 physics assumed for the interaction of bone and marrow. Elastic, viscoelastic, and fluid

46 constitutive properties have all been reported from experimental measurements of marrow

47 properties. It is unclear whether this ambiguity reflects the various length-scales, loading rates,

48 and boundary conditions of the experiments, or if the material models are sufficiently similar as

49 to be interchangeable. To address this question, we analyzed both the mean shear stress and its

50 spatial distribution induced in marrow during compression of trabecular bone cubes when using

51 linear elastic, neo-Hookean, viscoelastic, and power-law fluid constitutive models.

52 Experimentally reported parameters were initially applied for all four constitutive models,

53 resulting in poor agreement. The parameters of the soft solid models were calibrated by linear

54 interpolation so that the volume averaged shear stress agreed with the fluid model for each, but

55 this could only be accomplished on a specimen-by-specimen basis. Following calibration, the

56 root-mean-squared (RMS) difference between the solid and fluid constitutive models was still

57 greater than $26 \%$ even when the overall mean shear stress was in close agreement, indicating that

58 the spatial distribution of stress is also sensitive to the constitutive model. As such, the choice of

59 constitutive model should be backed by a strong rationale, and results should be interpreted with

60 care.

61 
62 Keywords: Bone marrow; Mechanobiology; Computational mechanics; Finite element

63 analysis; Fluid-structure-interaction; Constitutive model 


\section{Introduction}

65 Bone marrow is a complex, cellular tissue residing in the pore space of trabecular bone. It

66 is subject to cyclic motion as the bone deforms during activities of daily living. The resulting

67 mechanical deformation and stress may cause gene and protein expression in the various bone

68 marrow resident cell lineages, affecting immune and endocrine functions in addition to bone

69 adaption (Mantila Roosa et al., 2011; Soves et al., 2014). Due to the complex geometry of the

70 trabecular pores, understanding marrow mechanobiology is best approached by computational

71 models that couple the mechanics of both bone and marrow. Such models rely on implementing

72 appropriate constitutive models and boundary conditions.

73 Bone marrow has been characterized as a highly viscous fluid (Metzger et al., 2014), a

74 viscoelastic solid (Jansen et al., 2015), or a soft elastic solid (Shin et al., 2014; Winer et al.,

75 2009). These three constitutive models represent a range of computational complexity and

76 expense. Modeling bone marrow as a fluid requires the time-dependent Navier-Stokes equations

77 to be solved while coupled to the moving boundaries of the deforming solid in a fluid-structure-

78 interaction solution. In instances with a complex solid-fluid interface, like trabecular bone, a

79 fluid-structure interaction (FSI) simulation may be computationally prohibitive. In contrast, bone

80 marrow can be modeled as an elastic solid, allowing the solution to be computed in a single step

81 without time dependence. The viscoelastic assumption results in a solution with intermediate

82 expense, as the solution is time dependent, but coupling between marrow and bone is maintained

83 by the nodal connectivity.

84 It is common to apply simplified constitutive assumptions in computational models to

85 make problems more tractable. However, it is essential to understand how these assumptions

86 affect the model outcomes. Hence, the goal of this study was to compare the shear stress 
87 calculated in an in situ model of bone marrow using fluid, solid, and viscoelastic marrow

88 constitutive assumptions. Specifically, we compared both the mean and spatial difference in the

89 shear stress in bone marrow modeled as 1) a power-law fluid, 2) a linear elastic solid, 3) a neo-

90 Hookean solid, and a 4) viscoelastic solid during dynamic compression of human trabecular bone 91 samples.

\section{Methods}

93 Trabecular bone images from two human femurs obtained from a national donor bank

94 (National Disease Research Interchange) as part of a previous project were studied (Wu et al., 95 2013). Briefly, aligned cylindrical samples were prepared from the femoral neck (Wang et al., 96 2004; Wu et al., 2013). The samples were imaged by $\mu$-CT (Scanco $\mu \mathrm{CT}-80$, Brüttisellen,

97 Switzerland) at $20 \mu \mathrm{m}$ nominal resolution, with 500 projections at $70 \mathrm{kVp}, 120 \mathrm{~mA}$, with a 400

$98 \mathrm{~ms}$ integration time. A $3 \times 3 \times 3 \mathrm{~mm}$ cubic region of interest was selected for modeling. The two 99 samples had bone volume fractions of 0.40 and 0.09 .

100 The $\mu$-CT images were segmented into bone and marrow regions using a constant 101 threshold of $452 \mathrm{mg}-\mathrm{HA} / \mathrm{cc}$. Conformal tetrahedral finite element meshes were created using 102 BioMesh3D (SCIRun, Scientific Computing and Imaging Institute, University of Utah) with an 103 average element volume of $2000 \mu \mathrm{m}^{3}$. In a previous study, this mesh resolution resulted in 104 convergence of both the fluid and solid shear stress within less than 1\% (Metzger et al., 2015). 105 The bone was assigned a uniform isotropic modulus of $15 \mathrm{GPa}$ and Poisson's ratio of 0.3 106 (Bayraktar and Keaveny, 2004; Niebur et al., 2000). A sinusoidal strain profile was applied along 107 the inferior-superior axis of the bone by fixing the inferior nodes and uniformly displacing the 108 nodes on the superior surface (Fig. 1) to achieve a peak compressive strain of $1000 \mu$-strain in $1 / 2$ 
109 s. Four models were created for each mesh with a non-Newtonian fluid, a linear elastic solid, a

110 hyperelastic solid, and a viscoelastic solid constitutive model applied to the marrow region.

111 For the baseline case, the marrow was modeled as a non-Newtonian, homogenous fluid

112 (Bryant et al., 1989; Coughlin and Niebur, 2012; Dickerson et al., 2008; Metzger et al., 2015;

113 Ochoa et al., 1991). The bone marrow viscosity was assigned the power-law relationship

$114 \eta=\min \left(\mu_{0}, 85.5 \dot{\gamma}^{-1}\right)$, where $\eta$ is the viscosity $(\mathrm{Pa} \bullet \mathrm{s}), \dot{\gamma}$ is the shear rate $\left(\mathrm{s}^{-1}\right)$, and $\mu_{0}$ is an

115 upper bound on the viscosity (Metzger et al., 2014). Without such a limit, the initially static fluid

116 would have an infinite viscosity, and flow could not be initiated. The initial upper bound was

$1171000 \mathrm{~Pa} \bullet$ s, based on experimental reports. Subsequently, an upper bound of $100 \mathrm{~Pa} \bullet \mathrm{s}$ was applied

118 to better match the results of elastic simulations. A fluid density of $0.95 \mathrm{~g} / \mathrm{cm}^{3}$ was assigned

119 (Gurkan and Akkus, 2008). A no-slip interface was applied at the bone-marrow interface,

120 assuming that marrow cells attached to the bone matrix via integrins (Birmingham et al., 2014;

121 Dickerson et al., 2008; Metzger et al., 2015). This was the only kinematic boundary condition

122 assigned to the marrow, with free flow allowed at all six external surfaces. A zero pressure

123 boundary condition was assigned on the fluid face opposite the loaded surface (Fig. 1). The

124 resulting transient, dynamic FSI models were solved using an iterative coupling scheme in

125 ADINA 9.0.

126 To investigate the response of marrow when measured as a soft solid, three constitutive

127 models were considered: a linear elastic solid, a neo-Hookean hyperelatic solid, and a

128 viscoelastic solid. All properties were based on literature reports (Table 1). The marrow and bone

129 meshes were connected by equivalencing nodes at the interface. For the viscoelastic material

130 model, a single term Prony series with an instantaneous shear modulus of $2.5 \mathrm{kPa}$, a relaxed

131 modulus of $2 \mathrm{kPa}$, and a bulk modulus of $2.5 \mathrm{MPa}$ to approach incompressibility was applied 
132 (Jansen et al., 2015). Since the single term could not capture the reported complex modulus,

133 decay constants of $0.1,0.5$, and 1.0 were investigated to cover a range of behavior. The shear

134 modulus in the linear elastic and neo-Hookean models was further adjusted to minimize the error

135 in the mean shear stress between the solid and fluid simulations.

136 Finite element simulations were performed using ADINA® (Watertown, MA). The

137 elastic, hyperelastic and viscoelastic simulations were quasistatic. The solid phase of the FSI

138 model was quasistatic and the fluid phase was fully dynamic. Results were post-processed using

139 Paraview (Kitware, Clifton Park, NY). The data were quantified within a 2.5 x 2.5 x 2.5 mm sub-

140 region in the center of the sample to avoid edge effects. The volumetric mean shear stress was

141 calculated by integrating over the element volumes. To facilitate comparisons, the fluid model

142 was taken as baseline, and he difference of each elastic marrow model was compared to it.

143 Similarly, the spatial distribution in shear stress was compared by calculating the element by

144 element root-mean square (RMS) difference of each elastic marrow model from the fluid model.

\section{Results}

146 The solution time for the FSI simulations was nearly 50 times that of the elastic models.

147 Fluid structure interaction simulations required approximately 96 CPU hours and 15 GB of

148 RAM. The models employing solid constitutive models for marrow required only 2 CPU hours

149 and $2 \mathrm{~GB}$ of RAM. The fluid and viscoelastic simulations required multiple time steps because

150 of the time-dependency of the material model. The elastic models were solved in a single time

151 step, although the hyperelastic model required an iterative solution.

152 Compression of the bone induced shear stress in the marrow in both the solid and fluid

153 marrow models. However, the peak volume-averaged marrow shear stress occurred at the

154 maximum strain in the linear elastic, neo-Hookean, and viscoelastic models, while the fluid 
155 model reached its peak stress almost immediately upon loading and decreased to a minimum at

156 the maximum displacement. Altering the exponential parameter, $\alpha$, in the viscoelastic models

157 affected the results by less than $10 \%$. As such, only the results for the $1 \mathrm{~s}$ time constant are

158 presented.

159 When the bone marrow was modeled as a fluid, the upper bound viscosity increased the

160 marrow shear stress almost linearly, from an average of $0.42 \mathrm{~Pa}$ to $4.2 \mathrm{~Pa}$ for the $0.09 \mathrm{BV} / \mathrm{TV}$

161 specimen and from $0.58 \mathrm{~Pa}$ to $5.6 \mathrm{~Pa}$ in the $0.40 \mathrm{BV} / \mathrm{TV}$ specimen. The peak velocity gradient

162 magnitude was only $0.003 \mathrm{~s}^{-1}$, leaving most of the marrow within the regime of the upper bound.

163 The volume-averaged shear stress decreased with decreasing shear modulus in the elastic

164 material models (Fig. 2). The neo-Hookean model with $\mathrm{G}=220 \mathrm{~Pa}$ best approximated the

165 volume-averaged shear stress when compared to the $100 \mathrm{~Pa} \bullet \mathrm{s}-$ bound fluid model. The $2.5 \mathrm{kPa}$

166 viscoelastic model best approximated the volume-averaged shear stress when compared to the

$1671000 \mathrm{~Pa} \cdot$ s-bound fluid model.

168 The spatial distribution of shear stress varied significantly from the fluid simulations

169 (Fig. 3). The solid models under-predicted the shear stress at the bone-bone-marrow interface

170 when compared to the FSI simulations (Fig. 4).

171 The performance of the constitutive models was not consistent between the two samples

172 studied (Fig. 5A). The relative difference with the fluid model was four times higher in the high

173 volume fraction sample for the elastic and hyperelastic models, but had a similar magnitude for

174 the viscoelastic model (Fig. 5A). The $220 \mathrm{~Pa}$ linear elastic material model had the smallest RMS

175 difference from the fluid model when the limiting viscosity was $100 \mathrm{~Pa} \bullet \mathrm{s}$ in the $9 \%$ volume

176 fraction sample (Fig. 5B), while the $2.5 \mathrm{kPa}$ viscoelastic material model had the smallest

177 difference when compared to the $1000 \mathrm{~Pa} \bullet$ s fluid model (Fig. 5B). The RMS difference in the 
178 linear elastic and viscoelastic models was $24 \%$ and $14 \%$ higher in the high volume fraction

179 sample, respectively. In contrast, the RMS difference decreased by $6 \%$ between samples when

180 the neo-Hookean model was used (Fig. 5B).

\section{Discussion}

182 Computational models of bone marrow mechanics are important for addressing

183 hypotheses related to bone marrow mechanobiology. The reliability of such models depends on

184 choosing an appropriate constitutive model. We quantified the sensitivity of the computed stress

185 in trabecular bone marrow to the marrow constitutive model. For a single sample it was clear that

186 the constitutive parameters could be calibrated to match the fluid simulation. However, the

187 calibrated constitutive parameters depended on the sample, making it impractical to apply to

188 experimental data. Even when the solid constitutive model gave similar volume-averaged shear

189 stress within the marrow, the spatial distribution of stress exhibited substantial differences. The

190 RMS difference was more than $25 \%$ when compared to the fluid model, and was highest at the

191 bone surface where the highest mechanobiological signal would be expected. Finally, the elastic

192 models could not capture rate dependency, and the viscoelastic model did not have as rapid of a

193 decay in stress as the fluid model. As such, although marrow has been commonly modeled as a

194 soft solid to reduce computational expense, the assumption is not consistent with a fluid marrow.

195 The non-Newtonian fluid constitutive model was used as the baseline for comparison.

196 However, the true material properties of bone marrow in in vivo environments are not well

197 established. Bone marrow has been characterized as a power-law fluid (Bryant et al., 1989) with

198 properties similar to blood, a viscous power-law fluid (Metzger et al., 2014), a viscoelastic solid

199 (Jansen et al., 2015), a gel (Winer et al., 2009), or a linear elastic soft solid (Shin et al., 2014).

200 Although these different constitutive models could describe experimental results within 
201 reasonable error, our results demonstrate that these constitutive models differ substantially under

202 physiological conditions.

203 Bone marrow has been modeled as a linear-elastic solid (Webster et al., 2015) or a poro-

204 viscoelastic solid (Sandino et al., 2015) in mechanobiological studies. Our results indicate that

205 the distribution of shear stress within the marrow would differ substantially from fluid and

206 viscoelastic constitutive models based on recent experimental measurements (Jansen et al., 2015;

207 Metzger et al., 2014; Shin et al., 2014; Winer et al., 2009).

208 This study illustrates that the choice of constitutive model plays a critical role in

209 simulations of the biomechanics of the marrow niche in trabecular bone. Although modeling

210 bone marrow as a solid material decreases the overall computation time and memory required,

211 elastic models could not capture the spatial distribution of shear stress that results from assuming

212 fluid behavior. The results also differed substantially between elastic, hyperelastic, and

213 viscoelastic constitutive models, even when using the same modulus. Hence, the constitutive

214 model applied to marrow in computational studies should be critically evaluated based on the

215 outcome variables of interest, and should be consistent with experimental data.

\section{Acknowledgements:}

217 This research was supported by the U.S. National Science Foundation through grants CMMI

2181100207 and 1435467.

219 Conflict of Interest Statement

220 The authors have no conflicts of interest related to this publication. 


\section{References}

224 Bayraktar, H.H., Keaveny, T.M., 2004. Mechanisms of uniformity of yield strains for trabecular 225 bone. J. Biomech. 37, 1671-1678.

226 Birmingham, E.C., Kreipke, T.C., Dolan, E.B., Coughlin, T.R., Owens, P., McNamara, L.M., 227 Niebur, G.L., McHugh, P.E., 2014. Mechanical stimulation of bone marrow in situ induces bone formation in trabecular explants. Ann. Biomed. Eng. in press,.

229 Bryant, J.D., David, T., Gaskell, P.H., King, S., Lond, G., 1989. Rheology of bovine bone 230 marrow. Proc Inst Mech Eng H 203, 71-75.

231 Coughlin, T.R., Niebur, G.L., 2012. Fluid shear stress in trabecular bone marrow due to low232 magnitude high-frequency vibration. J. Biomech. 45, 2222-2229.

233 Dickerson, D.A., Sander, E.A., Nauman, E.A., 2008. Modeling the mechanical consequences of 234 vibratory loading in the vertebral body: microscale effects. Biomech Model Mechanobiol $235 \quad 7,191-202$.

236 Gurkan, U.A., Akkus, O., 2008. The mechanical environment of bone marrow: a review. Ann $237 \quad$ Biomed Eng 36, 1978-1991.

238 Isaksson, H., van Donkelaar, C.C., Ito, K., 2009. Sensitivity of tissue differentiation and bone 239 healing predictions to tissue properties. J. Biomech. 42, 555-564.

240 Jansen, L.E., Birch, N.P., Schiffman, J.D., Crosby, A.J., Peyton, S.R., 2015. Mechanics of intact 241 bone marrow. J Mech Behav Biomed Mater 50, 299-307.

242 Mantila Roosa, S.M., Liu, Y., Turner, C.H., 2011. Gene expression patterns in bone following 243 mechanical loading. J. Bone Miner. Res. 26, 100-112. 
244 Metzger, T.A., Kreipke, T.C., Vaughan, T.J., McNamara, L.M., Niebur, G.L., 2015. The in situ mechanics of trabecular bone marrow: the potential for mechanobiological response. J. Biomech. Eng. 137, 011006.

247 Metzger, T.A., Shudick, J.M., Seekell, R., Zhu, Y., Niebur, G.L., 2014. Rheological behavior of fresh bone marrow and the effects of storage. J Mech Behav Biomed Mater 40, 307-313.

249 Niebur, G.L., Feldstein, M.J., Yuen, J.C., Chen, T.J., Keaveny, T.M., 2000. High resolution 250 finite element models with tissue strength asymmetry accurately predict failure of trabecular bone. J. Biomech. 33, 1575-1583.

252 Ochoa, J.A., Heck, D.A., Brandt, K.D., Hillberry, B.M., 1991. The effect of intertrabecular fluid on femoral head mechanics. J. Rheumatol. 18, 580-584.

254 Sandino, C., McErlain, D.D., Schipilow, J., Boyd, S.K., 2015. The poro-viscoelastic properties of trabecular bone: a micro computed tomography-based finite element study. J Mech Behav Biomed Mater 44, 1-9.

Shin, J.W., Buxboim, A., Spinler, K.R., Swift, J., Christian, D.A., Hunter, C.A., Leon, C., Gachet, C., Dingal, P.C., Ivanovska, I.L., Rehfeldt, F., Chasis, J.A., Discher, D.E., 2014. Contractile forces sustain and polarize hematopoiesis from stem and progenitor cells. Cell

261 Soves, C.P., Miller, J.D., Begun, D.L., Taichman, R.S., Hankenson, K.D., Goldstein, S.A., 2014. Megakaryocytes are mechanically responsive and influence osteoblast proliferation and

264 Wang, X., Liu, X., Niebur, G.L., 2004. Preparation of on-axis cylindrical trabecular bone specimens using micro-CT imaging. J. Biomech. Eng. 126, 122-125. 
266 Webster, D., Schulte, F.A., Lambers, F.M., Kuhn, G., Muller, R., 2015. Strain energy density gradients in bone marrow predict osteoblast and osteoclast activity: A finite element study. J. Biomech. 48, 866-874.

269 Winer, J.P., Janmey, P.A., McCormick, M.E., Funaki, M., 2009. Bone marrow-derived human mesenchymal stem cells become quiescent on soft substrates but remain responsive to chemical or mechanical stimuli. Tissue Eng Part A 15, 147-154.

272 Wu, Z., Laneve, A.J., Niebur, G.L., 2013. In vivo microdamage is an indicator of susceptibility to initiation and propagation of microdamage in human femoral trabecular bone. Bone $55,208-215$. 
277 Table 1. Three solid constitutive models were investigated: linear elastic, neo-Hookean, and

278 viscoelastic. Material values were taken from the literature. Intermediate values were

279 interpolated based on the error in the mean shear stress from one of the fluid models. The

280 viscoelastic properties were based on the highest temperature tested in order to simulate

281 physiological behavior, and a bulk modulus of $2500 \mathrm{kPa}$ with a $10 \mathrm{~Pa}$ decay was applied to

282 approximate near incompressibility, and viscous behavior constrained to the deviatoric stress.

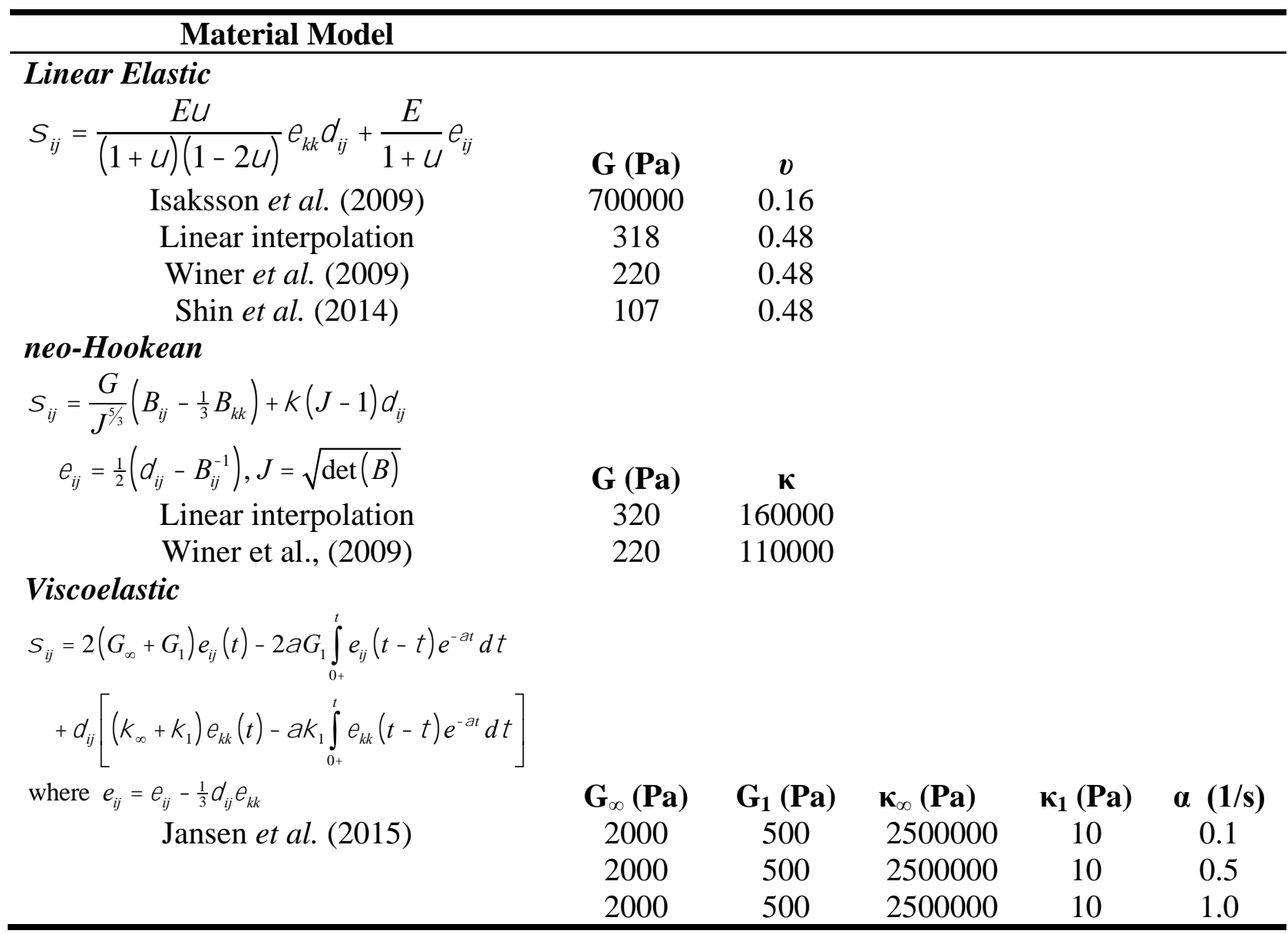




\section{Figure Captions}

286 Figure 1: A) Trabecular bone and marrow models were created from u-CT reconstructions and 287 meshed with BioMesh3D (SCIRun, University of Utah). B) Compressive strain was applied to

288 the superior surface of the bone models. C) For the FSI models the pressure was set to zero on 289 the inferior surface of the marrow. The only kinematic constraint on the marrow was a no-slip 290 boundary condition at the interface with the mineralized bone tissue.

292 Figure 2: The mean shear stress in the linear elastic and neo-Hookean models decreased

293 nonlinearly with decreasing shear modulus. The constitutive parameters were adjusted by linear

294 interpolation to reduce the average error. Elastic models with lower moduli were compared to the

295 fluid model with an upper bound viscosity of $100 \mathrm{~Pa} \bullet \mathrm{s}$ (A) while the elastic models with higher 296 moduli were compared to the $1000 \mathrm{~Pa} \bullet$ s bound power-law fluid model (B) in order to obtain the 297 best possible agreement.

299 Figure 3: The solid constitutive models resulted in higher stresses than the fluid models. The

300 locations of high stress and stress distributions differed between the elastic and fluid constitutive 301 models, as well as between the various elastic constitutive models.

303 Figure 4: The absolute value of the difference in shear stress between elastic and fluid 304 constitutive models exceeded $100 \%$ in many locations. A) Linear elastic, and B) neo-Hookean 305 compared to fluid with $\eta_{\max }=100$ Pa $\bullet$. C) Viscoelastic material compared to fluid with $\eta_{\max }=$ $3061000 \mathrm{~Pa} \bullet$ s. Note that the regions of highest error differ between all models, indicating that the 
307 three elastic constitutive models differ from one another as well as from the fluid constitutive

308 model.

309

310 Figure 5: A) When the same constitutive parameters were applied to samples of differing

311 volume fraction, the error in the mean shear stress differed sharply. As such, calibration of the

312 material properties is unlikely to be successful. B) The RMS difference from the fluid result was

313 also dependent on the sample analyzed, further indicating the differences in the constitutive

314 models. In both A) and B) the linear elastic and neo-Hookean materials were compared to the

$315100 \mathrm{~Pa} \bullet$ s upper bound power-law model, while the viscoelastic model was compared to the 1000

316 Pa•s upper bound power-law model.

317 
A
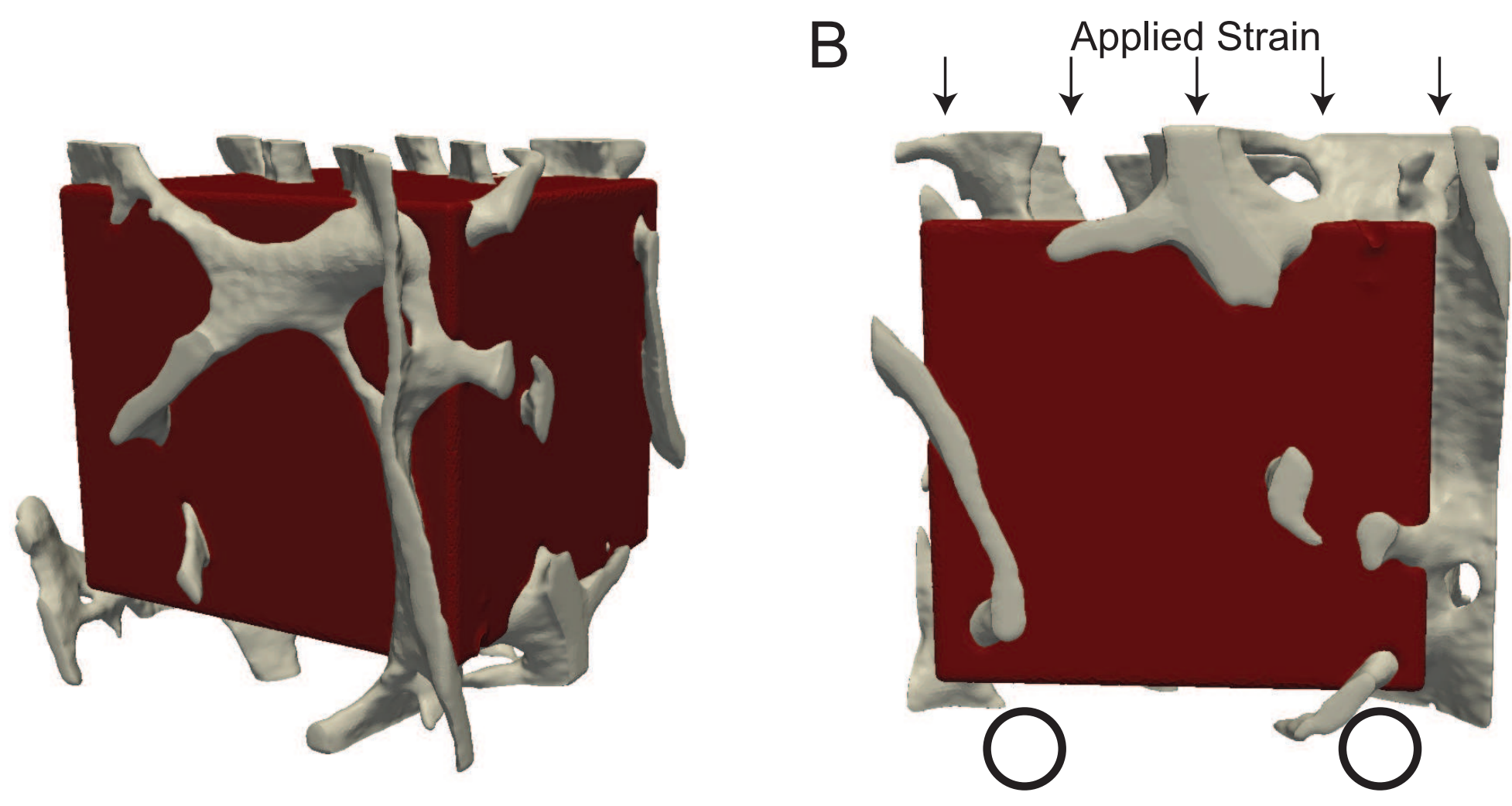

C

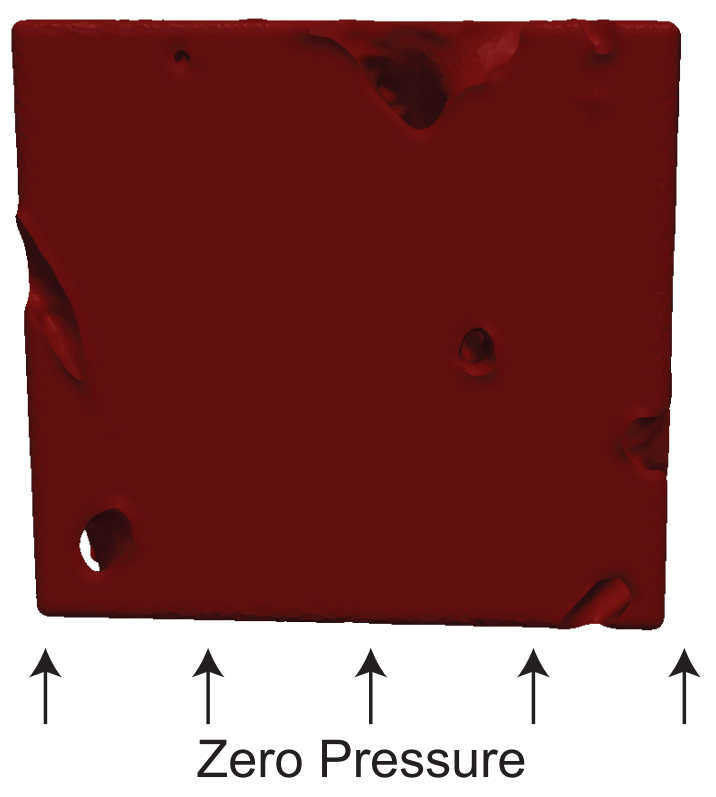




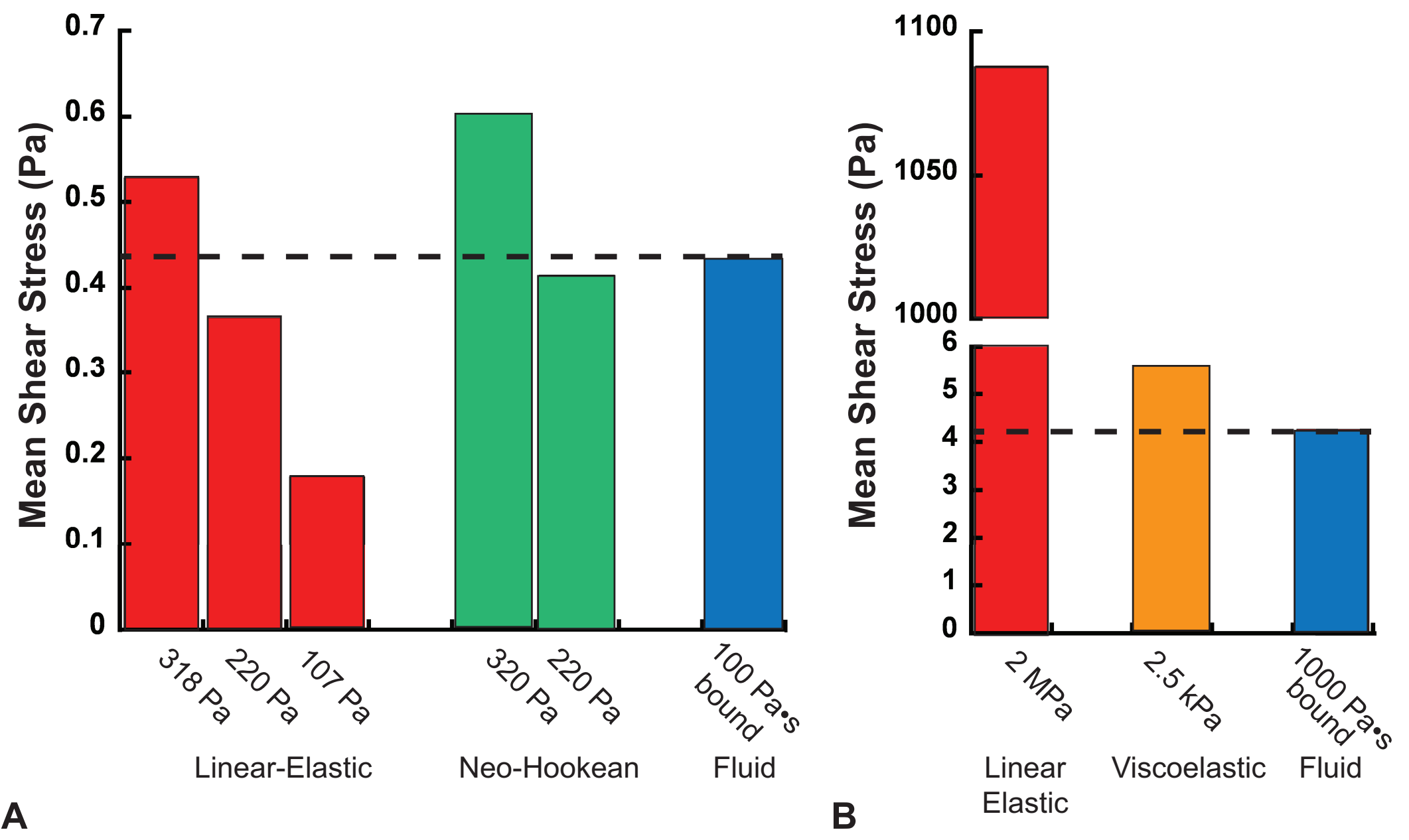



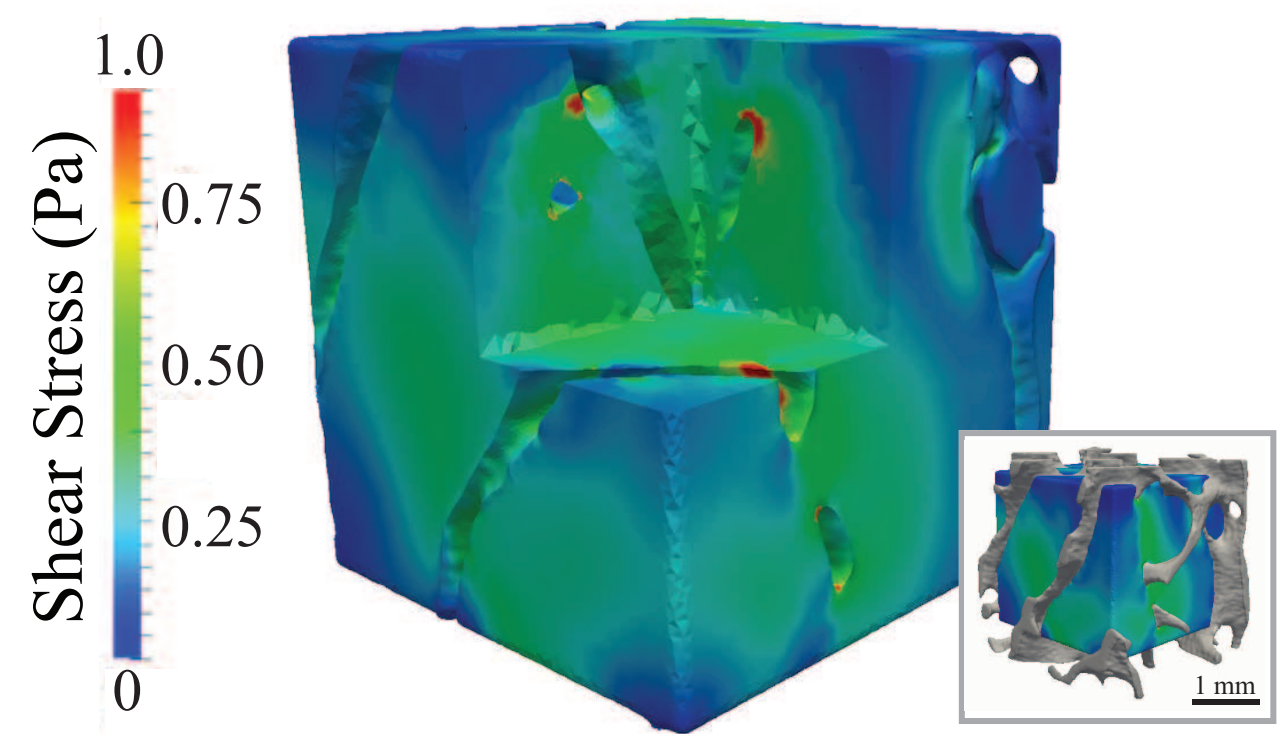

Power-Law

Bound=100 Pa॰s

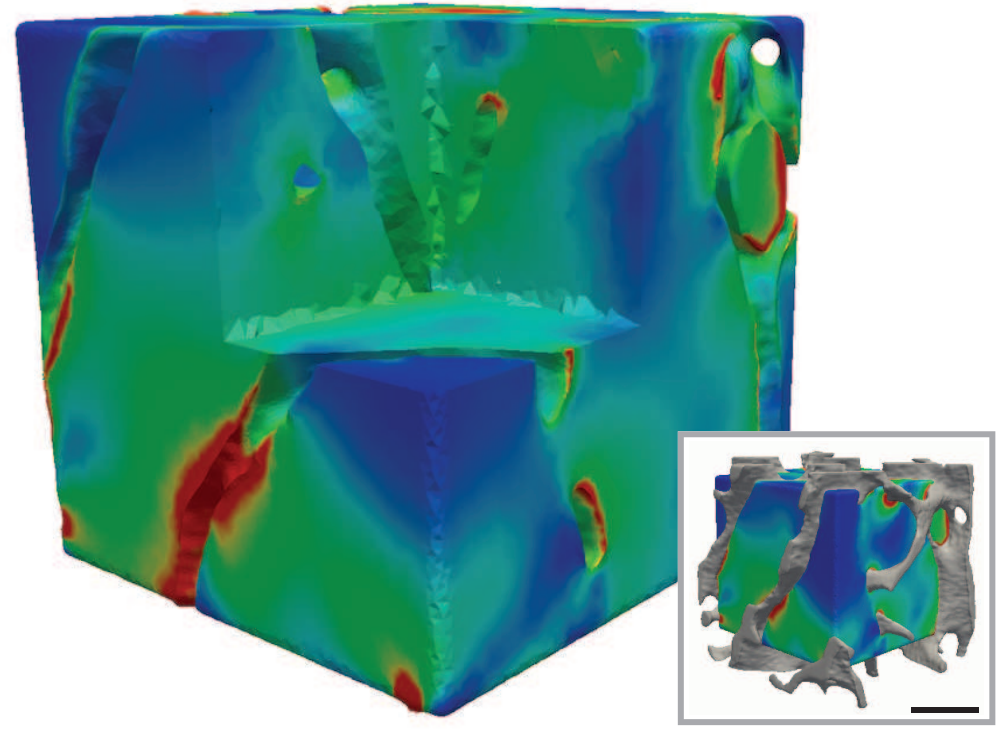

Linear Elastic

$\mathrm{G}=220 \mathrm{~Pa}$

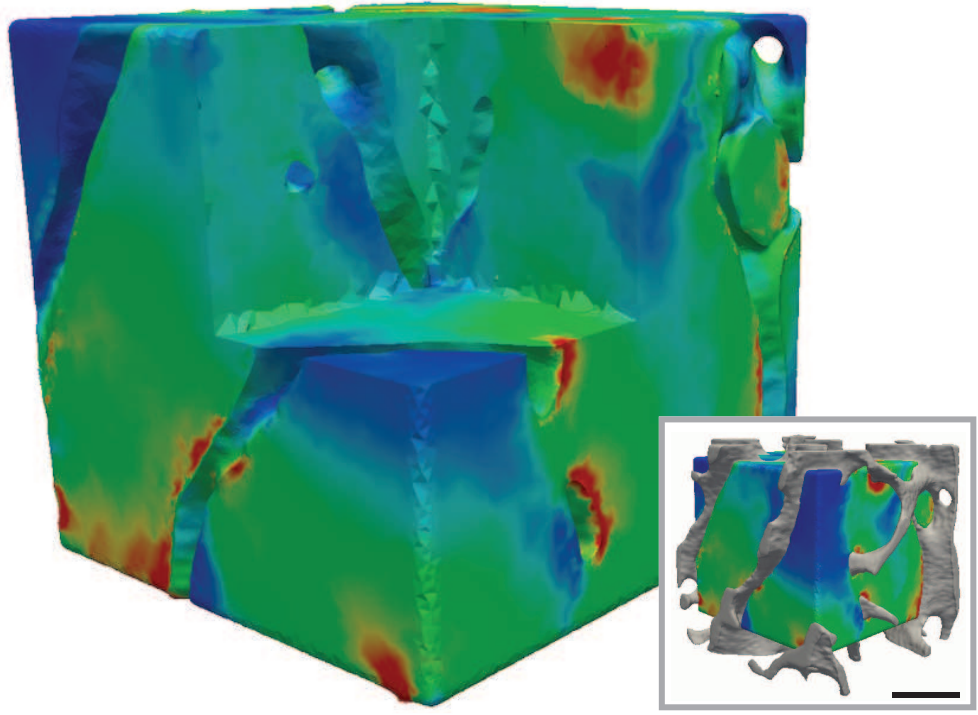

Neo-Hookean

$\mathrm{G}=220 \mathrm{~Pa}$ 
$A-100$
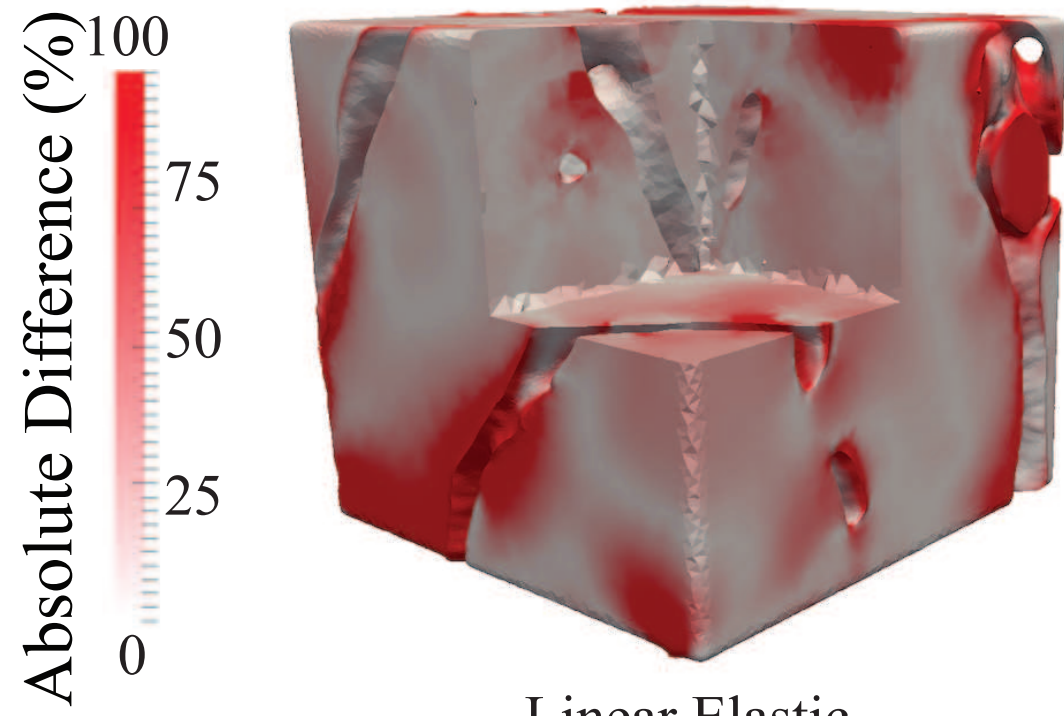

Linear Elastic $\mathrm{G}=220 \mathrm{~Pa}$

B

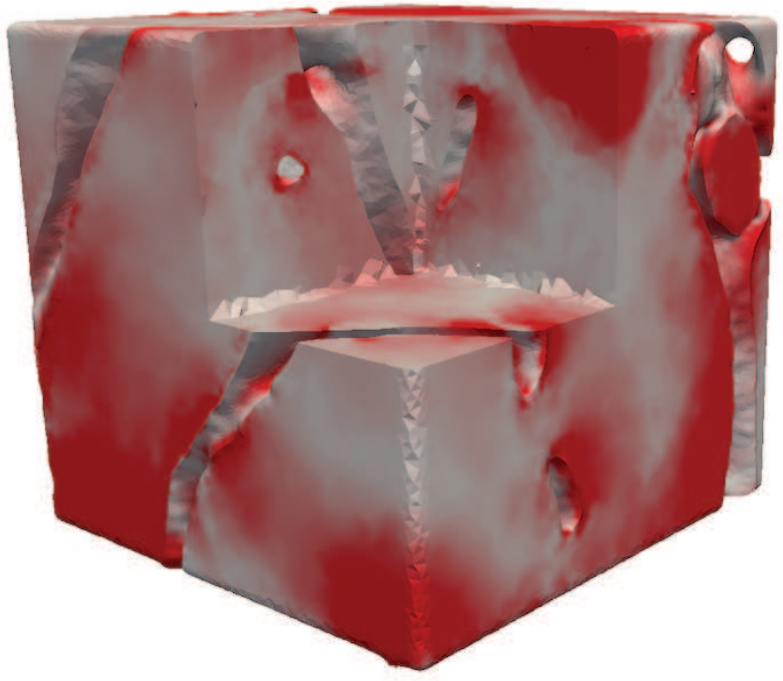

Neo-Hookean

$$
\mathrm{G}=220 \mathrm{~Pa}
$$

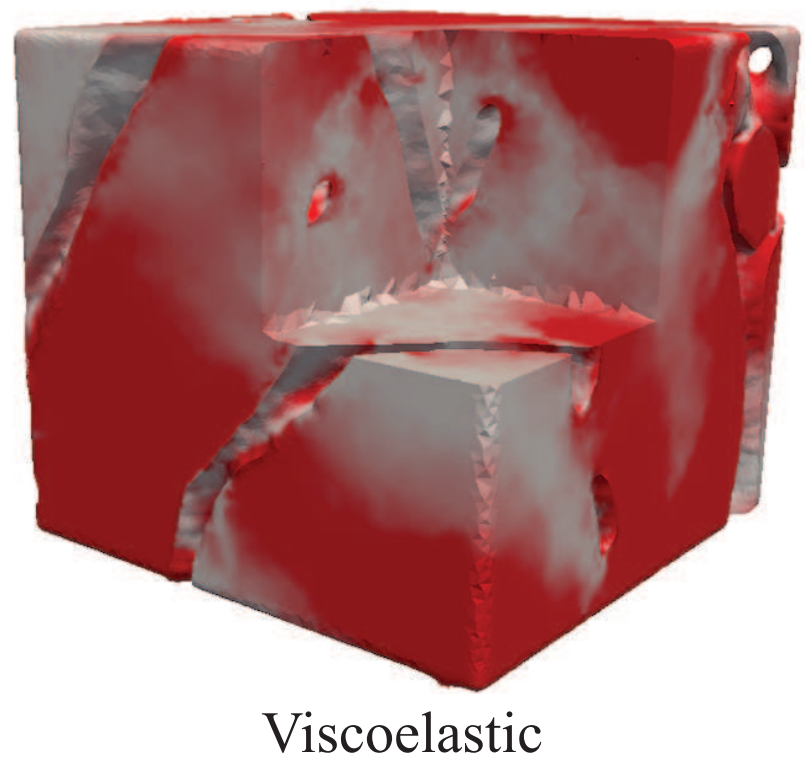

$\mathrm{G}=2.5 \mathrm{kPa}$ vs. $\eta_{\max }=1000 \mathrm{~Pa} \cdot \mathrm{s}$ 


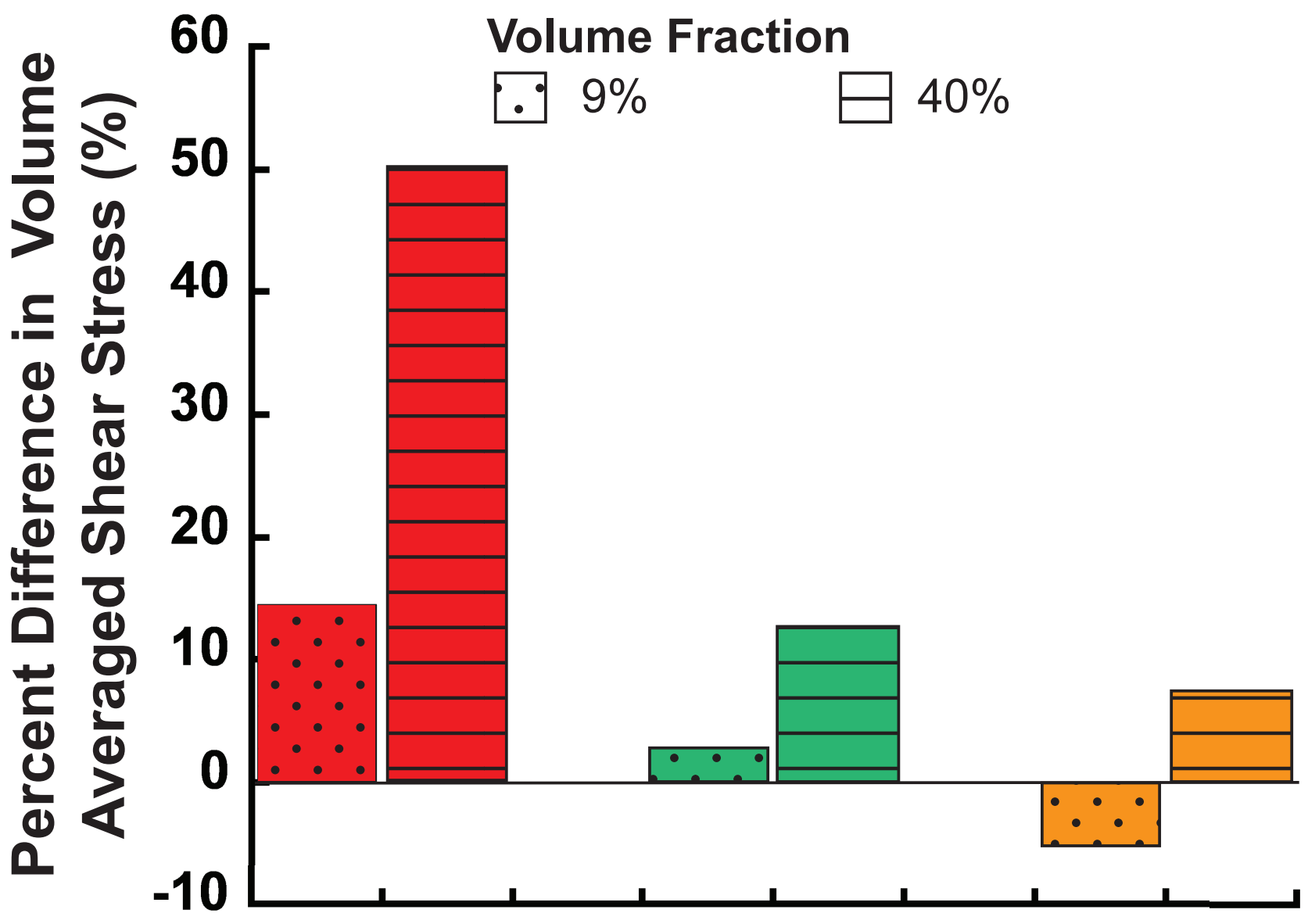

A

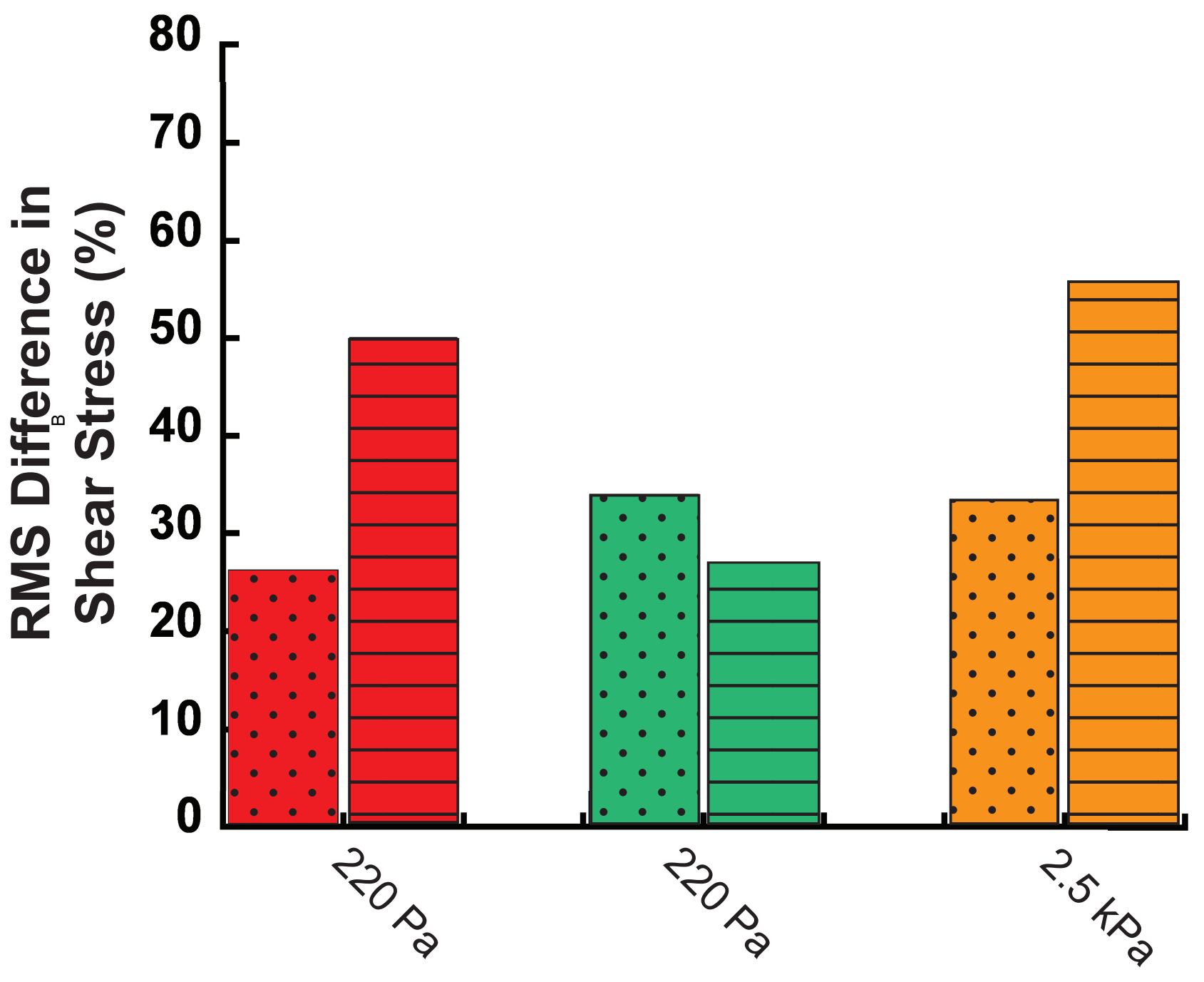

Linear Elastic Neo-Hookean

Viscoelastic 